\title{
Physical factors in the stimulation of colonic peristalsis
}

\author{
J. D. HARDCASTLE AND C. V. MANN \\ From St Mark's Hospital, London, and The London Hospital
}

SUMMARY The effect of colonic distension and changes in intraluminal $p \mathrm{H}$ and osmolality have been investigated in patients with well established colostomies. Small volumes of acidic buffer $(p \mathrm{H} 4)$ and hypertonic saline $(4 \%)$ were found to be only a very weak peristaltic stimulus. Strong hypertonic solutions (glycerine BP) induced a poorly sustained peristaltic response in the majority of cases. Distension of the inactive colon did not stimulate peristalsis. Distension of a colon previously stimulated to peristaltic activity caused fresh waves of contraction. This study appears to show that by themselves physical factors play an unimportant role in initiating peristaltic activity in the colon.

Colonic peristalsis can be stimulated by the use of surface-acting agents such as bisacodyl and oxyphenisatin (Hardcastle and Mann, 1968). Little is known, however, of the factors which initiate spontaneous peristalsis.

This study was undertaken to investigate the role of colonic distension and changes of intraluminal $p \mathrm{H}$ and osmolality in the stimulation of peristalsis.

\section{Methods}

Intraluminal colonic pressures were recorded using small air-filled latex balloons $(0.4 \times 0.8 \mathrm{~cm})$ introduced into the bowel through well established colostomies, in a manner similar to that previously described (Hardcastle and Mann, 1968).

Only healthy bowel has been studied. The transverse colostomies were performed to defunction the bowel after resection of carcinoma of the rectum and the sigmoid colostomies were established after excision of the rectum for carcinoma. Only one study was performed on each patient (Table I).

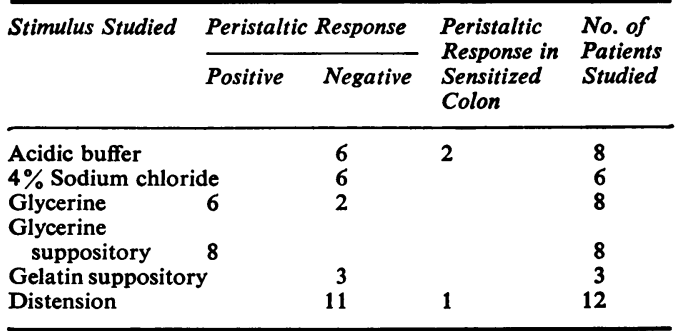

Table I Summary of responses to various stimuli

\section{Results}

EFFECT OF CHANGE OF INTRALUMINAL $p H$ The effect of introducing 4-6 $\mathrm{ml}$ of phosphate buffer, $p \mathrm{H} 4$, into the lumen of the colon was studied in eight patients. On six occasions no change in bowel activity was noted (Fig. 1) although the colon responded by peristalsis to subsequent intraluminal bisacodyl. On two occasions, poorly sustained peristalsis was noted, but on one of these, peristalsis had already been observed before the introduction of the acidic buffer solution (Fig. 2). 


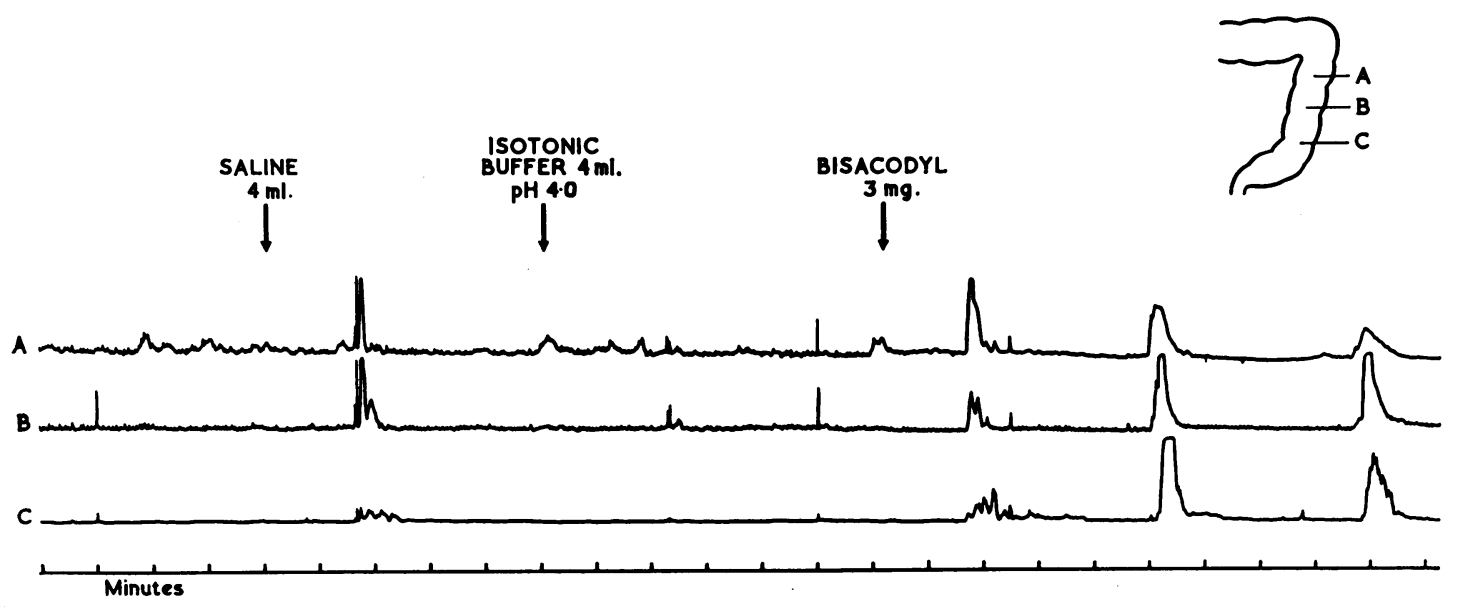

Fig. 1 Effect of acid buffer on the colon.

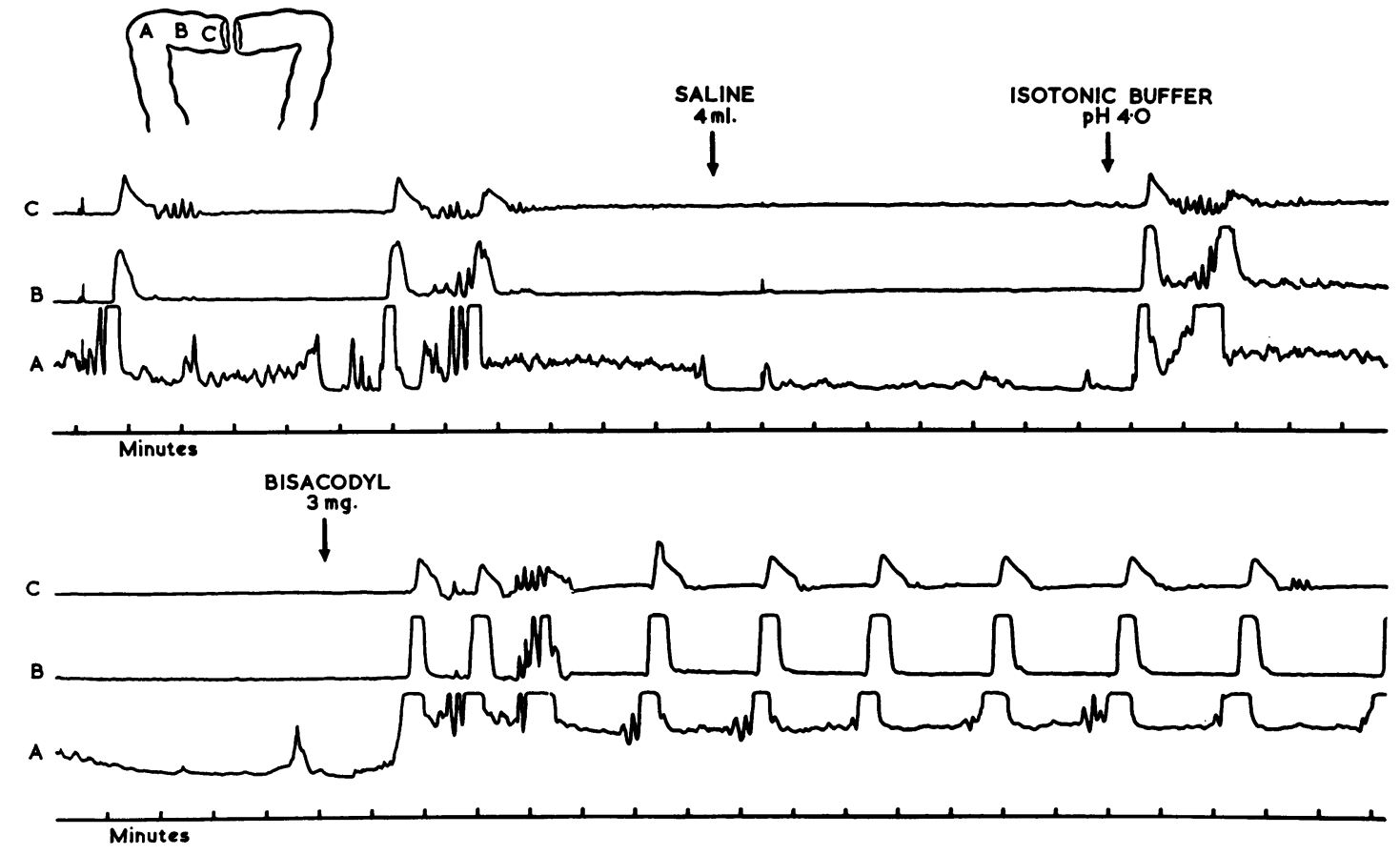

Fig. 2 Effect of acidic solutions on colon previously sensitized to peristaltic activity. 


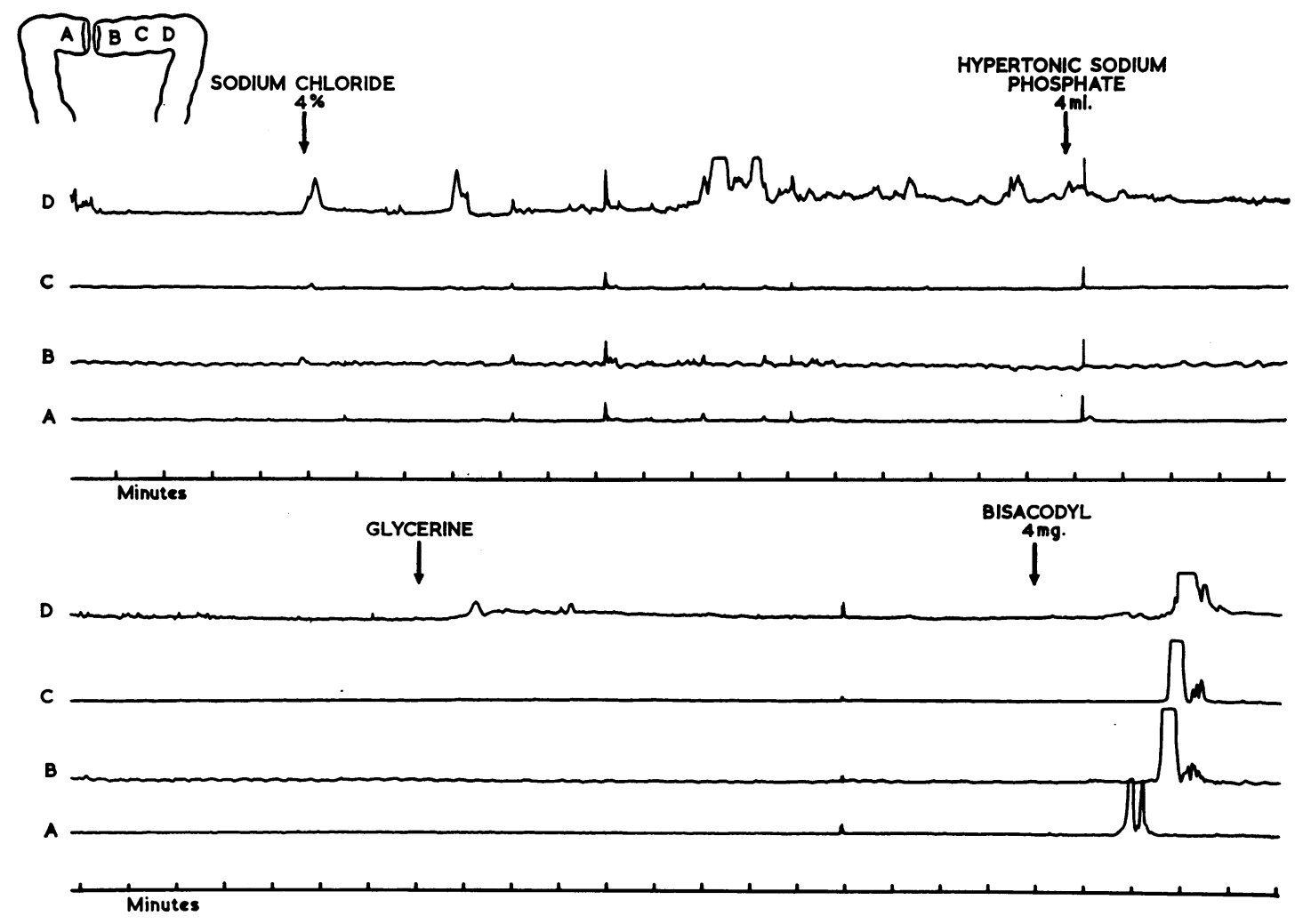

Fig. 3 Effect of hypertonic solutions on the colon.

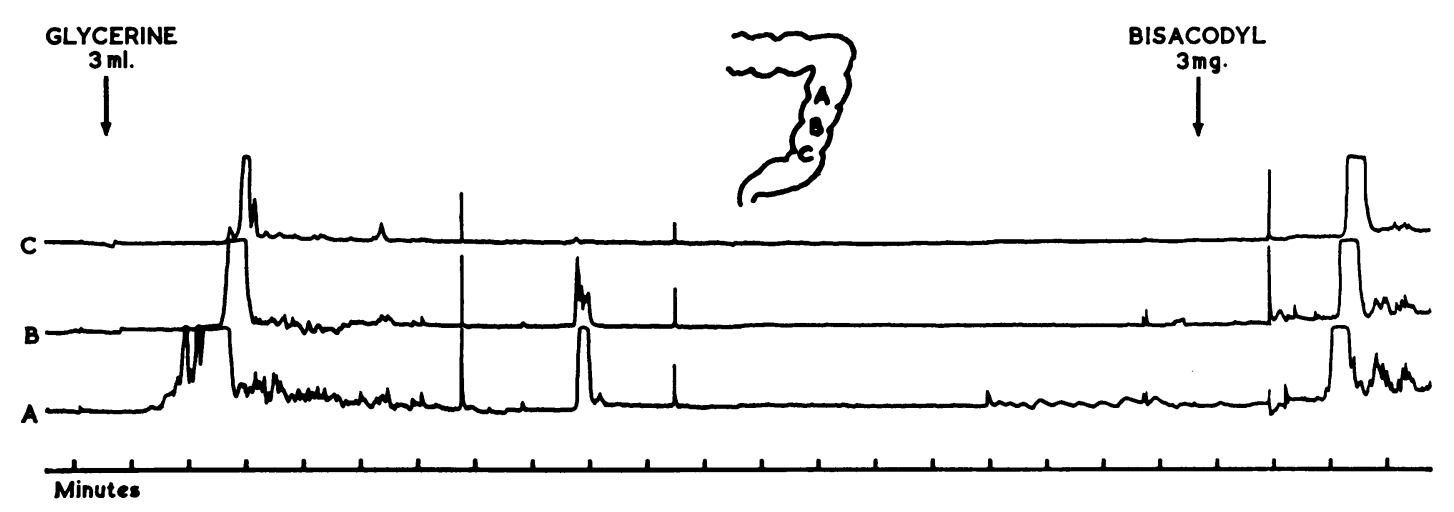

Fig. 4 Effect of glycerine on the colon. 


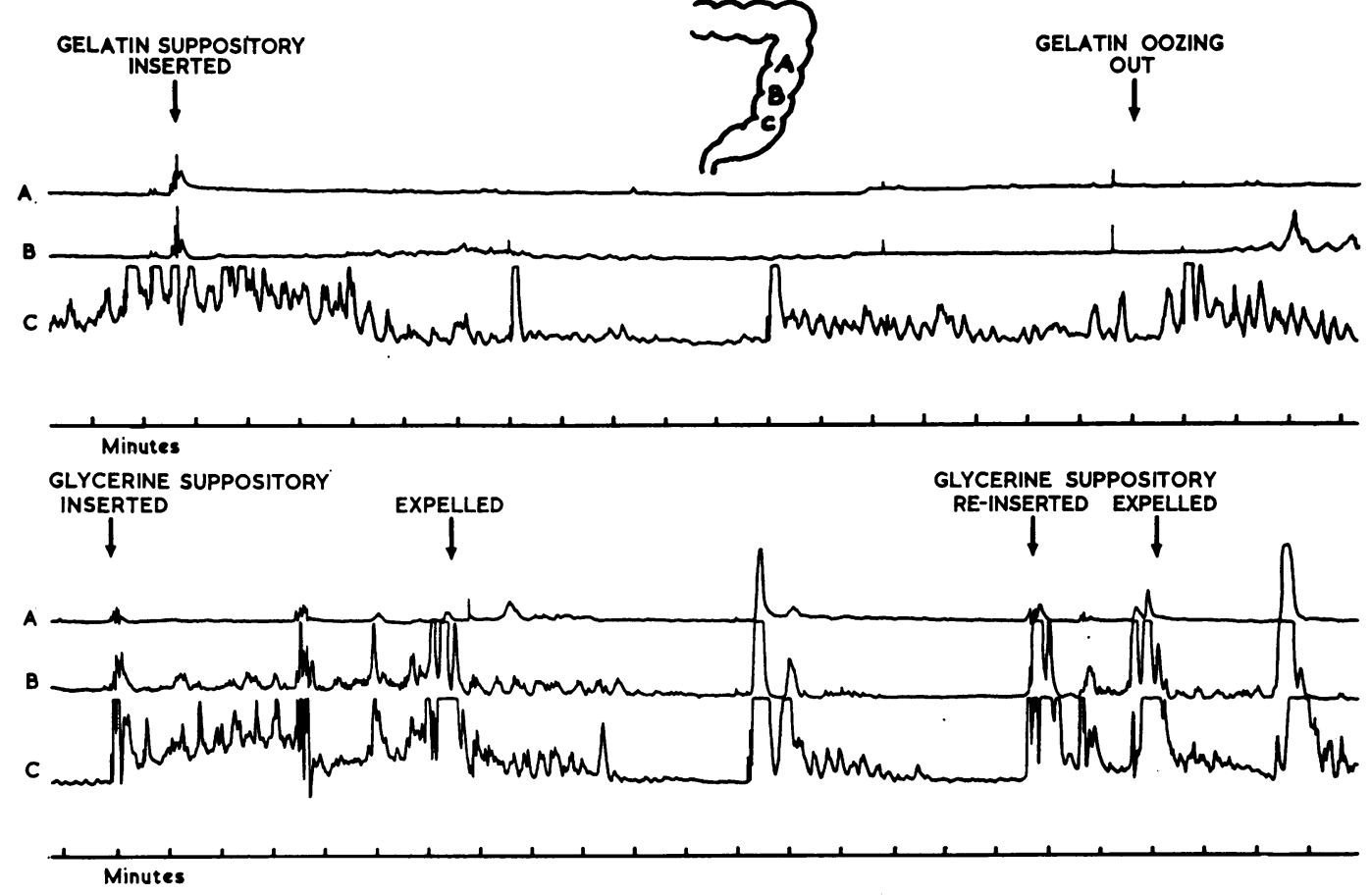

Fig. 5 Effect of gelatin and glycerine suppositories on the colon.

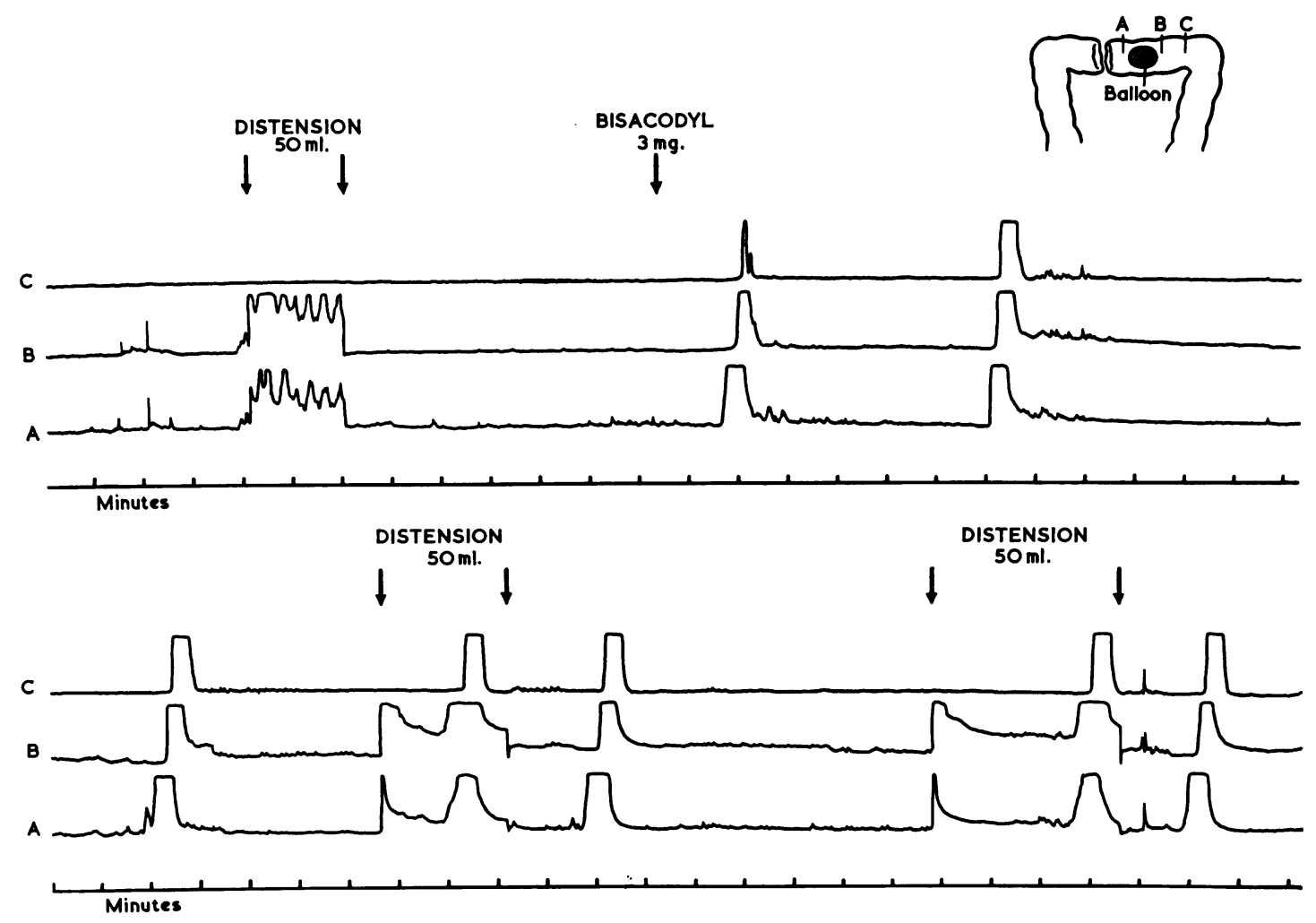

Fig. 6 Effect of distension on the colon. 


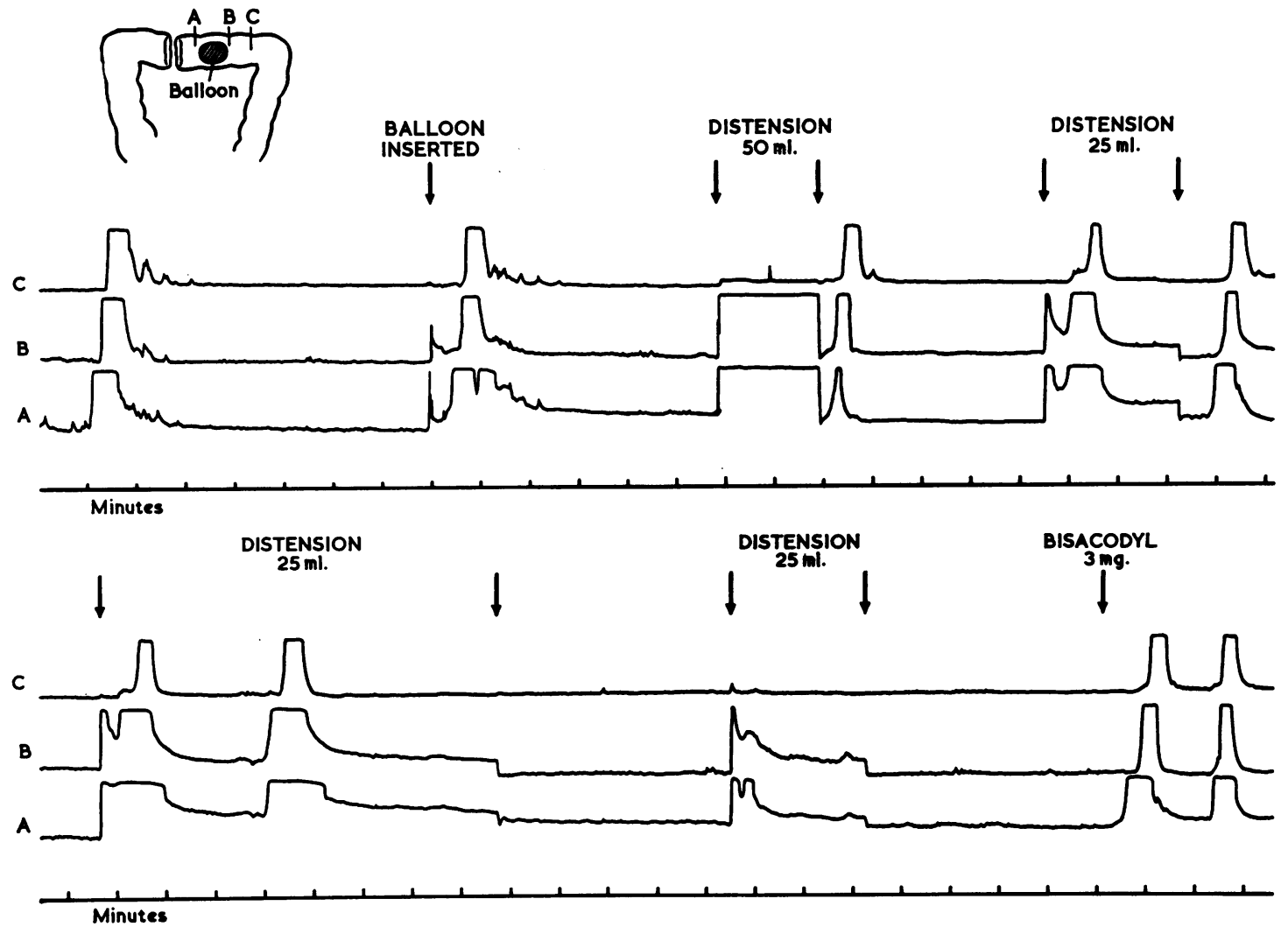

Fig. 7 Effect of distension on colon previously sensitized to peristaltic activity.

EFFECT OF CHANGE OF INTRALUMINAL OSMOLALITY

The effect of intraluminal $4 \%$ sodium chloride $(4-8 \mathrm{ml})$ was studied in six patients. No propagated activity was observed, although local increase in activity was seen (Fig. 3). Intraluminal glycerine $(3 \mathrm{ml})$ stimulated a poorly sustained peristaltic response in six subjects (Fig. 4); no response was noted in two other patients (Fig. 3). The introduction of glycerine suppositories into the colon caused limited peristaltic activity which usually resulted in expulsion of the suppository from the colostomy. Suppositories made only of gelatine and water did not stimulate peristalsis (Fig. 5).

EFFECT OF DISTENSION OF THE COLON The effect of distension of the colon has been investigated in 12 patients using rapid distension of a thin latex balloon with $50 \mathrm{ml}$ of air as the distending stimulus.

In 11 patients, peristalsis was not stimulated (Fig. 6). In the study illustrated in Fig. 7, peristalsis was observed during the control period of observation; in this case insertion of the latex ballon and its subsequent inflation stimulated waves of peristalsis. In all cases after peristalsis had been induced by bisacodyl, further waves of contraction could be induced by distension (Fig. 6).

\section{Discussion}

In isolated colonic loops $\mathrm{N} / 100$ hydrochloric acid has been shown to increase the rate of movement of fluid through the bowel (Hukuhara, Nakayama, and Nanba, 1961). The $p H$ of the buffer solution used in this study was maintained at $4 \cdot 0$, a level well below the normal $p H$ of the contents of the colon (Kitagawa, Nishigori, Murata, Nishimoto, and Takada, 1966) and the volumes used were so small that significant distension was not produced. Acidic fluid used in this fashion proved to be only a very weak peristaltic stimulus.

Small volumes of $4 \%$ sodium chloride did not stimulate colonic peristalsis; as the ionic content of this solution is greatly in excess of that found in the terminal ileum (Kanaghinis, Lubran, and Coghill, 1963), it seems unlikely that changes in osmolality stimulate spontaneous peristalsis. However, extremely hypertonic solutions such as glycerine stimulate a poorly sustained peristaltic response and this would appear to be the mech- 
anism by which glycerine suppositories exert their effect.

The colon has been shown to accept a distending balloon with only slight increases in local activity unless the bowel has been previously sensitized either through endogenous factors or by the use of exogenous stimulants such as Bisacodyl.

Physical stimuli would thus appear to play little part in stimulating spontaneous peristalsis in man but may be important in creating further waves of contraction in bowel that has already been conditioned by other factors in the ileal dejecta.

We should like to thank Dr E. N. Rowlands and the MRC Gastroenterological Research Unit at the Central Middlesex Hospital for providing the Schwarzer pressure recording apparatus used in this and the previous study, and Boehringer Ingelheim Ltd for providing a supply of Bisacodyl (Dalcolox).
References

Hardcastle, J. D., and Mann, C. V. (1968). Study of large bowel peristalsis. Gut, 9, 512-520.

Hukuhara, T., Nakayama, S., and Nanba, R. (1961). The role of the intrinsic mucosal reflex in the fluid transport through the denervated colonic loop. Jap. J. Physiol., 11, 71-79.

Kanaghinis, T., Lubran, M., and Coghill, N. F. (1963). The composition of ileostomy fluid. Gut, 4, 322-338.

Kitagawa, K., Nishigori, A., Murata, N., Nishimoto, K., and Takada, H. (1966). Radiotelemetry of the $p H$ of the gastrointestinal tract by glass electrode. Gastroenterology, 51, 368-372. 